\section{Actividad física y calidad de vida en personas con enfermedad renal crónica}

\author{
YISEL PINILLOS-PATIÑO ${ }^{1, \mathrm{a}, \mathrm{b}}$, YANETH HERAZO-BELTRÁN ${ }^{1, \mathrm{a}, \mathrm{b}}$, \\ JORGE GIL CATAÑO ${ }^{2, \mathrm{c}, \mathrm{d}}$, JOHANNA RAMOS DE ÁVILA ${ }^{1, \mathrm{a}, \mathrm{d}}$
}

\section{Association of physical activity with quality of life in patients with chronic kidney disease}

Background: Physical activity may improve quality of life in patients with chronic kidney disease. Aim: To assess the relationship between physical activity and quality of life in patients with Chronic Kidney Disease. Material and Methods: The Kidney Disease Quality of Life-36 (KDQOL-36) and the International Physical Activity questionnaire were answered by 130 patients with chronic kidney disease ( 74 women, 80 receiving renal substitution therapy). Sociodemographic variables were recorded. Results: Patients on renal substitution therapy with a time lapse since diagnosis of 0 to 6 months had higher levels of physical activity than those with longer time lapses (51.4 \pm 12.5 and $34.6 \pm 8.1$ minutes respectively). Disease burden scores were lower among patients with renal substitution therapy. There was a direct correlation between levels of vigorous and moderate physical activity and the physical functioning dimension in the quality of life questionnaire for patients with more than 19 months of disease. The dimension general physical health was significantly associated with physical activity in women and patients with 7 to 18 months of diagnosis. The dimension disease burden was associated with physical activity in women, patients not receiving substitution therapy and those with 7 to 18 months of diagnosis. Conclusions: Moderate and vigorous physical activity is directly related to the dimensions physical functioning, the general perception of physical health and inversely related with the dimension burden of disease.

(Rev Med Chile 2019; 147: 153-160)

Key words: Exercise; Kidney Failure, Chronic; Quality of Life; Renal Dialysis.

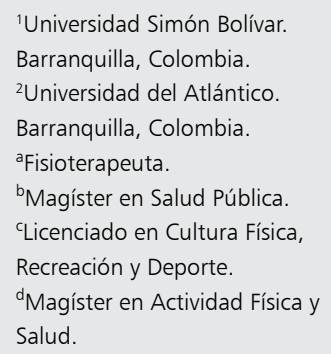

Declaración sobre conflictos de intereses: Los autores declaran que no existe conflicto de intereses de tipo económico o de otra índole con persona natural o institucional.

Recibido el 1 de junio de 2018, aceptado el 23 de enero de 2019.

\section{Correspondencia a:} Yisel Pinillos-Patiño Carrera 59No. 59-65 ypinillos@unisimonbolivar.edu.co

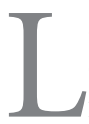

a enfermedad renal crónica (ERC) se define como la función renal alterada que se confirma en dos o más ocasiones con al menos 3 meses de diferencia ${ }^{1}$, es progresiva, no transmisible, silenciosa y de alto costo ${ }^{2,3}$, debido al incremento en las tasas de morbilidad y mortalidad, llegando a considerarse la epidemia del siglo 21 y un problema de salud pública mundial afectando a $35 \%$ de los mayores de 70 años ${ }^{4}$.

La prevalencia en España es de 9,16\% ${ }^{5}$ y en los Estados Unidos de Norteamérica, entre 2011 y 2014, se estimó una prevalencia de $14,8 \%{ }^{6}$.
Para Latinoamérica, se ha encontrado reportes de México, donde la incidencia es de $10 \%$, con mayor afectación en adultos ${ }^{7}$. En Chile, $2,7 \%$ de su población la padece y la tendencia al incremento se observa en el número de pacientes hemodializados $^{8}$. La prevalencia en Colombia es de 2,8\% ${ }^{4}$ con tendencia al incremento, comportamiento similar al resto de los países, lo cual se evidencia en reportes de cerca de 23.578 personas tratados con terapia dialítica, 16.634 en hemodiálisis y 6.844 en diálisis peritoneal ${ }^{9}$.

Las personas en terapia de reemplazo o tra- 
tamiento sustitutivo, generalmente presentan disminución de la capacidad cardiorrespiratoria, desnutrición, fatiga y pérdida de la masa muscular, que junto con enfermedades crónicas concomitantes llegan a afectar la capacidad funcional del individuo ${ }^{10}$. El bajo rendimiento que resulta de un consumo menor de oxígeno y el cambio morfológico muscular genera alteraciones en las dimensiones personal, social, familiar y laboral con importante repercusión sobre la calidad de vida relacionada con la salud $(\text { CVRS })^{11}$, resultados que han demostrado ser adecuados para predecir la mortalidad y hospitalización ${ }^{12,13}$.

El ejercicio físico, además de aumentar la fuerza muscular, prevenir la pérdida de masa muscular, incrementar la función cardiovascular y optimizar la supervivencia al reducir el riesgo cardiovascular, mejora la CVRS de los pacientes con $\mathrm{ERC}^{10}$. Este importante aspecto depende no solo de un estilo físicamente activo, sino del tiempo transcurrido desde el inicio del tratamiento ${ }^{14}$ y del tipo de tratamiento, dado que las terapias sustitutivas, además de eliminar los desechos del metabolismo que son dañinos para la salud, repercuten en los componentes psicológicos, socioeconómicos y espiritual que rodean a quienes son diagnosticados con $\mathrm{ERC}^{15}$.

Resulta interesante identificar la percepción de cada individuo sobre su situación de vida dentro del contexto cultural en el que se desarrolla, y la relación con una vida activa, debido a que una baja calidad de vida $(\mathrm{CV})$ representa un riesgo de 1,7 veces mayor para tener una tasa de filtración glomerular disminuida ${ }^{13,16}$. La actividad física puede impactar en general en las personas con ERC debido a los beneficios que se muestran tanto en la salud física como en la mental y emocional en quienes se mantienen activos ${ }^{17,18}$.

Pocos estudios han evaluado el impacto potencial de la actividad física (AF) y la CV en personas con un nivel reducido de la función renal, y al ser la ERC considerada de alto costo, en la que se ha introducido nuevos factores de riesgo emanados del consumo, la pobreza, la inequidad social y de salud, este aspecto se torna importante dada la tasa de supervivencia de los pacientes. La CVRS es una medida de resultado clave y la actividad física una herramienta que favorece el ajuste de los pacientes a las limitaciones físicas, los cambios en el estilo de vida y los tratamientos médicos ${ }^{16}$. Por lo anterior, se propuso con este trabajo establecer la relación entre la AF y la percepción de la CVRS en personas con ERC.

\section{Material y Método}

Estudio de corte transversal en pacientes adultos con ERC que asisten a una institución prestadora de servicio (IPS) de tercer nivel de la ciudad de Barranquilla, Colombia. Mediante muestreo no probabilístico e intencional, se seleccionó 130 pacientes con ERC de todos los que asistieron a la consulta por nefrología y a la unidad de diálisis durante los 4 meses del estudio. De 200 pacientes, se excluyó 70 ( 15 por deterioro cognitivo y 55 no aceptaron participar). Las características sociodemográficas fueron evaluadas mediante una encuesta que indagó sobre el sexo, estado civil, estrato socioeconómico, procedencia, escolaridad y nivel de aseguramiento, tiempo de diagnóstico y si recibía tratamiento sustitutivo.

La CVRS se evaluó con el cuestionario Kidney Disease Quality of Life-36 (KDQOL-36), el cual ha sido adaptado culturalmente en pacientes colombianos con ERC terminal ${ }^{20}$. Este instrumento permite identificar el funcionamiento $y$ bienestar de los sujetos y mide dos componentes: el genérico (SF-12) que valora funcionamiento físico, desempeño físico, dolor físico, salud general, desempeño emocional, funcionamiento social, vitalidad y salud mental y el componente específico (KDQOL-36), que mide los síntomas y problemas, la carga y efectos de la enfermedad en la vida diaria ${ }^{19}$. Cada dimensión se puntúa entre 0 a 100, y los valores más altos reflejan mejor CVRS.

El Cuestionario Internacional de Actividad Física (IPAQ) formato corto permitió estimar el promedio del tiempo semanal (expresado en minutos) de actividad física de moderada a vigorosa en una semana $(\mathrm{AFMV})^{21}$. De acuerdo con el IPAQ formato corto, el punto de corte para definir una persona activa o inactiva es 150 min de actividad física de intensidad moderada o 75 min de intensidad vigorosa. Todos los participantes fueron informados sobre los procedimientos y objetivos del estudio, respetando las normas éticas concordantes con la Declaración de Helsinki, previa aprobación por el Comité de Ética la Universidad Simón Bolívar.

El software estadístico SPSS versión 24 permitió el análisis de los datos. Para cada variable cuantitativa se estimó un valor promedio y su desvia- 
ción estándar y para las categóricas se presenta la frecuencia absoluta y relativa. Se realizó prueba de Kolmogorov-Smirnov (K-S) para verificar la normalidad de los datos, y se realizó la prueba $U$ de Mann-Whitney para la diferencia de medias de las dimensiones de calidad de vida según el sexo y el tratamiento sustitutivo. Las diferencias de medias de las dimensiones de calidad de vida según el tiempo de diagnóstico de la enfermedad se definieron mediante la prueba Kruskal Wallis. Se determinó la relación entre las dimensiones de CVRS y los minutos de AFMV semanal mediante el coeficiente de Spearman en los pacientes que recibían o no tratamiento sustitutivo y en los que tenían 0-6 meses, 7-18 meses y más de 19 meses de diagnóstico de ERC. El nivel de significancia estadística se determinó en $p \leq 0,003$, dado el número de comparaciones realizadas entre las variables.

\section{Resultados}

En la Tabla 1 se presentan las características sociodemográficas de la población estudiada. El $56,9 \%$ (74/130) de los participantes fueron mujeres. En las características clínicas se evidencia que $37,7 \%$ (49/130) tenía entre 7 y 18 meses de diagnóstico y $61,5 \%(80 / 130)$ seguía un tratamiento sustitutivo (Tabla 2).

La Tabla 3 muestra diferencias significativas ( $\mathrm{p} \leq 0,003)$ entre los minutos de la combinación de AFMV realizados semanalmente y si los pacientes recibían o no tratamiento sustitutivo; fue mayor la práctica de AF en personas que recibían tratamiento sustitutivo y en quienes tenían de 0 a 6 meses de diagnóstico de ERC, con medias de $51,4 \pm 12,5 \mathrm{~min}$ y $34,6 \pm 8,1 \mathrm{~min}$.

Para las dimensiones del componente genérico de CV no se encontró diferencias entre hombres y mujeres. Quienes no recibían tratamiento sustitutivo tenían puntajes promedios más altos en la dimensión carga de la enfermedad renal, representado en $78,8 \pm 14,1$ y los que recibían alcanzaron 70,5 $\pm 14,6(\mathrm{p} \leq 0,003)$; la dimensión efectos de la enfermedad en la vida diaria obtuvo mayor puntaje en los que no recibían tratamiento sustitutivo, 30,7 $\pm 13,9$, a diferencia de los que se trataban, 22,2 $\pm 9,8(\mathrm{p} \leq 0,003)$. Aunque no se encontraron diferencias significativas en los puntajes promedios de las dimensiones de $\mathrm{CV}$ de acuerdo con el tiempo de diagnóstico de ERC, la salud física fue mayor en sujetos con menor tiempo de diagnóstico, 59,8 $\pm 14,9$, comparado con los de mayor tiempo, 56,8 $\pm 15,2$ (Tabla 3 ).

La práctica de AFMV se relacionó directamente con el funcionamiento físico en las personas con más de 19 meses de haber sido diagnosticadas

\section{Tabla 1. Caracterización de los pacientes según} variables sociodemográficas

\begin{tabular}{|c|c|c|}
\hline Característica & Frecuencia & $\%$ \\
\hline \multicolumn{3}{|l|}{ Sexo } \\
\hline Mujer & 74 & 56,9 \\
\hline Hombre & 56 & 43,1 \\
\hline \multicolumn{3}{|l|}{ Estado civil } \\
\hline Soltero & 23 & 17,7 \\
\hline Casado & 88 & 67,7 \\
\hline Separado o divorciado & 8 & 6,2 \\
\hline Viuda o viudo & 11 & 8,5 \\
\hline \multicolumn{3}{|l|}{ Estrato socioeconómico } \\
\hline Estrato 1 & 80 & 61,5 \\
\hline Estrato 2 & 34 & 26,2 \\
\hline Estrato 3 & 10 & 7,7 \\
\hline Estrato 4 & 3 & 2,3 \\
\hline Estrato 5 & 1 & 0,8 \\
\hline Estrato 6 & 2 & 1,5 \\
\hline \multicolumn{3}{|l|}{ Procedencia } \\
\hline Urbano & 106 & 81,5 \\
\hline Rural & 24 & 18,5 \\
\hline \multicolumn{3}{|l|}{ Escolaridad } \\
\hline Primaria & 73 & 56,2 \\
\hline Secundaria & 40 & 30,8 \\
\hline Técnico & 9 & 6,9 \\
\hline Estudios superiores & 8 & 6,2 \\
\hline \multicolumn{3}{|l|}{ Nivel de aseguramiento } \\
\hline Contributivo & 48 & 36,9 \\
\hline Subsidiado & 78 & 60 \\
\hline Vinculado & 4 & 3,1 \\
\hline
\end{tabular}

Tabla 2. Características clínicas de los pacientes renales participantes

\begin{tabular}{|lcc|}
\hline & Frecuencia & \% \\
\hline Tiempo diagnóstico & & \\
0 a 6 meses & 38 & 29,2 \\
7 a 18 meses & 49 & 37,7 \\
Más de 19 meses & 43 & 33,1 \\
Tratamiento sustitutivo & & \\
No & 50 & 38,5 \\
Sí & 80 & 61,5 \\
\hline
\end{tabular}




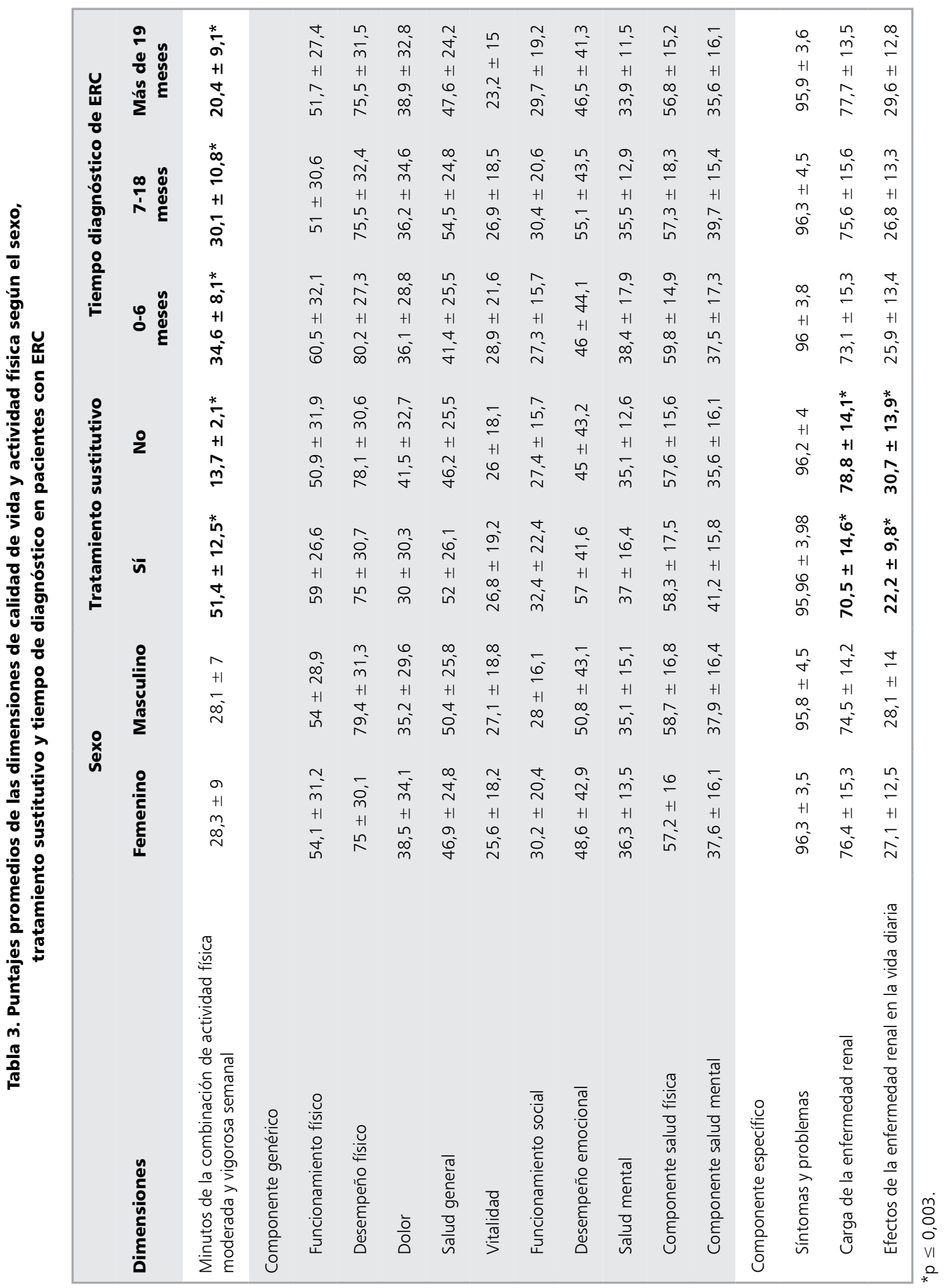


Tabla 4. Coeficientes de correlación entre las dimensiones de calidad de vida y los minutos de práctica de actividad física moderada y vigorosa según el sexo, tratamiento sustitutivo y tiempo de diagnóstico en pacientes con ERC

\begin{tabular}{|c|c|c|c|c|c|c|c|}
\hline \multirow[b]{2}{*}{ Dimensiones } & \multicolumn{2}{|c|}{ Sexo } & \multicolumn{2}{|c|}{ Tratamiento sustitutivo } & \multicolumn{3}{|c|}{ Tiempo diagnóstico de ERC } \\
\hline & $\begin{array}{c}\text { Feme- } \\
\text { nino }\end{array}$ & $\begin{array}{l}\text { Mas- } \\
\text { culino }\end{array}$ & Sí & No & $\begin{array}{c}0-6 \\
\text { meses }\end{array}$ & $\begin{array}{c}\text { 7-18 } \\
\text { meses }\end{array}$ & $\begin{array}{l}\text { Más de } \\
19 \text { meses }\end{array}$ \\
\hline \multicolumn{8}{|l|}{ Componente genérico } \\
\hline Funcionamiento físico & 0,16 & 0,13 & 0,24 & 0,16 & 0,017 & 0,20 & $0,36^{*}$ \\
\hline Desempeño físico & 0,29 & 0,10 & 0,30 & 0,13 & 0,07 & 0,32 & 0,27 \\
\hline Dolor & 0,08 & 0,04 & 0,05 & 0,07 & 0,06 & 0,12 & 0,04 \\
\hline Salud general & 0,10 & 0,23 & 0,05 & 0,17 & 0,33 & 0,18 & 0,17 \\
\hline Vitalidad & 0,10 & 0,08 & 0,09 & 0,15 & 0,09 & 0,13 & 0,09 \\
\hline Funcionamiento social & 0,06 & 0,09 & 0,22 & 0,11 & 0,13 & 0,15 & 0,04 \\
\hline Desempeño emocional & 0,06 & 0,018 & 0,13 & 0,05 & 0,16 & 0,06 & 0,13 \\
\hline Salud mental & 0,26 & 0,17 & 0,25 & 0,25 & 0,10 & 0,36 & 0,03 \\
\hline Componente salud física & $0,34^{*}$ & 0,08 & 0,30 & 0,09 & 0,06 & $0,37^{*}$ & 0,09 \\
\hline Componente salud mental & 0,10 & 0,09 & 0,06 & 0,08 & 0,10 & 0,12 & 0,14 \\
\hline \multicolumn{8}{|l|}{ Componente específico } \\
\hline Síntomas y problemas & 0,08 & 0,13 & 0,20 & 0,09 & 0,13 & 0,08 & 0,23 \\
\hline Carga de la enfermedad renal & $0,34^{*}$ & 0,04 & 0,22 & $0,40 *$ & 0,08 & $0,42 *$ & 0,27 \\
\hline $\begin{array}{l}\text { Efectos de la enfermedad renal } \\
\text { en la vida diaria }\end{array}$ & 0,20 & 0,02 & 0,17 & 0,08 & 0,07 & 0,03 & 0,18 \\
\hline
\end{tabular}

${ }^{*} p \leq 0,003$.

$(\mathrm{r}=0,36)$, con el componente general salud física en mujeres $(r=0,34)$ y en pacientes con tiempo de diagnóstico entre 7 y 18 meses $(r=0,37)$. Igualmente, con la carga de la enfermedad renal en mujeres $(r=0,34)$, en quienes no usan tratamiento sustitutivo $(r=0,40)$ y en pacientes con tiempo de diagnóstico entre 7 a 18 meses $(r=0,42)$ $(\mathrm{p} \leq 0,003)$ (Tabla 4$)$.

\section{Discusión}

Analizando la variable calidad de vida se puede decir que es útil para distinguir diferencias entre pacientes y definir de esta manera intervenciones centradas en las necesidades y características identificadas, de tal manera que favorezcan la recuperación de la capacidad funcional de las personas con ERC. En este sentido, programas que estimulen la práctica de actividad física de estas personas, se convierte en un objetivo primordial, teniendo en cuenta los beneficios que brinda tanto a nivel fisiológico, funcional y psicológico, aspectos fundamentales en la atención de quienes padecen esta enfermedad y además se enfrentan a tratamientos sustitutivos.

Los resultados del presente estudio se corresponden con los hallazgos de Barros y cols. ${ }^{22}$, así como lo reportado por Seguí y cols. ${ }^{23}$ quienes informaron como características de las personas observadas mayor predominio de hombres $53,1 \%$ frente a mujeres $46,9 \%$ con padecimiento de ERC, al igual que el estar casado como el estado civil más frecuente en ellos; lo anterior indica que son mujeres casadas las que con mayor frecuencia se encuentran en los estudios de personas con ERC, importante característica para la toma de decisiones en la definición de objetivos de intervención que incluyan a su núcleo familiar. En este sentido el tiempo de diagnóstico y las patologías asociadas se considera determinante para la definición de un programa, frente a ello Barros ${ }^{22}$ encontró diagnósticos de más de 37 meses con tratamiento de hemodiálisis; y reportó que 91,3\% tenía patologías asociadas a la enfermedad renal crónica y más del $80 \%$ con factores de riesgos asociados. Lo anterior se relaciona con este estudio en torno al tiempo de diagnóstico de los pacientes observados; sin 
embargo, no con la presencia de patologías asociadas las cuales no se observaron en los pacientes de este informe.

Respecto a la práctica de actividad física, se observó mejores resultados en quienes reciben tratamiento sustitutivo y quienes tienen menor tiempo de diagnóstico de la enfermedad. De acuerdo con lo anterior, Hernández ${ }^{24}$ indicó que, de 82 pacientes con tratamiento de hemodiálisis, 80 personas resultaron sedentarias. Reporte que coincide con Shimoda y cols ${ }^{25}$, quienes encontraron que a menor práctica de $\mathrm{AF}$, mayores reportes de muertes, $34 \%$ de los sujetos, en comparación con el grupo más activo $(19,6 \%)$; indicó que $24,5 \%$ de los pacientes al iniciar el tratamiento de hemodiálisis experimentan una disminución en la $\mathrm{AF}$, mientras que $26,6 \%$ indicaron un aumento de $30 \%$ en esta práctica. La inactividad física podría contribuir a incrementar la tasa de mortalidad en pacientes tratados con hemodiálisis, dado que las personas con ERC suelen ser más inactivos que aquellos que tienen una función normal del riñón ${ }^{25}$, aun cuando en una etapa media de la enfermedad se logre mejorar estos niveles, pero vuelven a disminuir con la cronicidad de la misma; la función física reducida se convierte en un predictor de morbilidad cardiovascular y mortalidad en ERC, porque la disminución de la $\mathrm{AF}$ a lo largo del tiempo es considerada de mal pronóstico.

Los hallazgos relacionados con el género y la CV no presentaron diferencias significativas según el estudio de Sánchez y cols ${ }^{26}$, lo cual concuerda con el reporte de este estudio. Respecto a la administración de tratamiento, Rebollo y cols ${ }^{13}$, indican que la CVRS se ve afectada en pacientes que reciben tratamiento sustitutivo; entre tanto que Sánchez y cols $^{26}$ concuerdan en que los mejores puntajes de sus participantes fue en el dominio psicológico para quienes recibían tratamiento sustitutivo; sin embargo, reportaron que los participantes tuvieron menor puntaje en el domino físico, siendo el aspecto que más se encuentra afectado en las personas con ERC en estadío terminal y bajo tratamiento de hemodiálisis; lo anterior difiere con lo reportado en este estudio, por lo tanto, es posible indicar que la salud mental es mejor en aquellos que son tratados con terapia sustitutiva ya que los efectos de la enfermedad son de mayor impacto en quienes no la reciben; entre tanto, la salud física es mejor percibida en los inicios de la enfermedad y con el tiempo esta percepción se ve afectada debido al deterioro y las alteraciones que se evidencian a pesar de la terapia sustitutiva lo cual se estima podría mejorar con la actividad física.

Qiu y cols ${ }^{27}$ reportaron que la AF tiene beneficios entre las personas enfermas y mejora el consumo máximo de oxígeno, por lo tanto, el ejercicio físico puede ayudar a los pacientes en su función física y capacidad aeróbica restaurando su funcionamiento. Broers y cols ${ }^{28}$ indicaron que los parámetros de AF fueron más bajos en pacientes con tiempo de diálisis menor o igual a 6 meses, lo cual es un rango distintivo de inactividad física; observaron cambios en el gasto energético relacionado con la actividad en los primeros seis meses de haber iniciado el tratamiento de diálisis y la velocidad para caminar aumentó de manera significativa con el paso del tiempo. Esto permite indicar la necesidad de intervenciones de entrenamiento físico conjuntas al tratamiento sustitutivo, para incrementar los parámetros de AF antes y después de iniciar la diálisis.

Rogan y cols $^{29}$ mostraron que los pacientes con ERC reportaron menor puntuación para los dominios individuales de la puntuación mental; lo anterior es importante, ya que los trastornos depresivos son los problemas de salud mental más comunes en quienes son sometidos a hemodiálisis, dada las diversas restricciones a las que deben someterse, así como a las modificaciones significativas en sus relaciones con el entorno inmediato, su capacidad para el desempeño social, familiar, laboral y general ${ }^{30}$.

Sobre la carga de la enfermedad, Capote y $\operatorname{cols}^{31}$ indicaron puntuaciones más bajas para los sujetos observados; se puede indicar que la calidad de vida tiene relación con el tratamiento sustitutivo, y el mayor impacto que se genera en la calidad de vida es en la dimensión carga de la enfermedad; es posible inferir que los determinantes de la carga de la enfermedad y la salud en general tienen influencia sobre la CV de una persona con ERC con tratamiento sustitutivo, dado el impacto sobre su funcionamiento físico y mental, esto repercute en su precepción relacionado con el bienestar y la habilidad para funcionar productivamente en la vida cotidiana.

Se puede concluir que la ERC tiene efectos negativos sobre la $\mathrm{CV}$ y la funcionalidad, y aunque los tratamientos sustitutivos generan alteraciones, 
quienes lo reciben tienen mejores puntajes promedios en el componente de salud mental. En la medida que aumenta el tiempo de diagnóstico y no se recibe tratamiento suele presentarse disminución en la práctica de AF, expresado como una disminución de su funcionalidad. Esto es relevante para el diseño de programas de intervención integrales que no solo busquen prolongar la vida, sino mejorar la calidad de vida referido a mejor salud en general, lo cual se considera una herramienta efectiva para mejorar la CV en las dimensiones de salud mental, salud física, desempeño emocional, desempeño físico, vitalidad y percepción de síntomas/problemas y, por ende, su integración social al mejorar la autopercepción ${ }^{22}$.

Como aspecto positivo de este estudio se considera el análisis entre la AF y la CVRS en pacientes con ERC, permitiendo observar el comportamiento de estas dos variables consideradas determinantes en el abordaje integral de personas con este tipo de diagnóstico. Por lo demás, el método utilizado se corresponde con el objetivo trazado, mas no orienta la determinación de las causas de los resultados, lo cual es motivo de otro estudio que permita estimar la relación causal entre las variables $\mathrm{CV}$ y $\mathrm{AF}$.

Agradecimientos: Los autores agradecen a los pacientes de la institución prestadora de servicios de salud participantes en la investigación, así como a la maestría en Actividad Física y Salud de la Universidad Simón Bolívar por brindar el acompañamiento en este trabajo.

\section{Referencias}

1. Gansevoort RT, Correa-Rotter R, Hemmelgarn BR, Jafar TH, Heerspink HJ, Mann JF, et al. Chronic kidney disease and cardiovascular risk: epidemiology, mechanisms, and prevention. Lancet 2013; 382 (9889): 339-52.

2. Lopera MM. La enfermedad renal crónica en Colombia: necesidades en salud y respuesta del Sistema General de Seguridad Social en Salud. Rev. Gerenc. Polít. Salud. 2016; 15 (30): 212-33.

3. Acuña L, Sánchez P, Soler LA, Alvis LF. Enfermedad renal crónica en Colombia: prioridad para la gestión de riesgo. Rev Panam Salud Publica 2016; 40 (1): 16-22.

4. Hill NR, Fatoba ST, Oke JL, Hirst JA, O’Callaghan CA, Lasserson DS, et al. Global Prevalence of Chronic Kidney Disease-A Systematic Review and Meta-Analysis.
PLoS One 2016; 11 (7): e0158765. doi: 10.1371/journal. pone. 0158765 .

5. Dorado DA, Estébanez ÁC, Pérez PM. Características de los pacientes registrados con enfermedad renal crónica en Castilla y León y análisis de supervivencia de los trasplantados y de sus injertos. Nefrología 2011; 31 (5): 579-86.

6. Saran R, Robinson B, Abbott KC, Agodoa LY, Albertus P, Ayanian J, et al. US Renal Data System 2016 Annual Data Report: Epidemiology of Kidney Disease in the United States. Am J Kidney Dis 2017; 69 (3 Suppl 1): A7-A8.

7. Gámez AM, Montell OA, Quintero V, Alfonso De León J, Hay De La Puente M. Enfermedad renal crónica en el adulto mayor. Rev Méd Electrón 2013; 35 (4): 306-18.

8. Zúñiga C, Muller OH, Flores M. Prevalencia de enfermedad renal crónica en centros urbanos de atención primaria. Rev Med Chile 2011 (9): 1176-84.

9. Cuenta de Alto Costo. Fondo Colombiano de Enfermedades de Alto Costo. Situación de la enfermedad renal crónica en Colombia. Bogotá: COLGRAF Editores; 2015. Disponible en: https://cuentadealtocosto.org/ site/images/Publicaciones/Situacion $\% 20 \mathrm{de} \% 201 \mathrm{a} \% 20$ ERC\%20Colombia\%202014.pdf.

10. Hernández A, Monguí K, Rojas Y. Descripción de la composición corporal, fuerza muscular y actividad física en pacientes con insuficiencia renal crónica en hemodiálisis en una unidad renal en Bogotá, Colombia. Rev Andal Med Deporte 2018; 11 (2): 52-56.

11. Canney M, Sexton E, Tobin K, Kenny RA, Little MA, O'Seaghdha CM. The relationship between kidney function and quality of life among community-dwelling adults varies by age and filtration marker. Clin Kidney J 2018; 11 (2): 259-64.

12. Ruiz P, Gómez PM, Crespo GM, Tierno TC, Crespo MR. Análisis de la calidad de vida del paciente en la etapa prediálisis. Enferm Nefrol 2017; 20 (3): 233-40.

13. Hall RK, Luciano A, Pieper C, Colón-Emeric CS. Association of Kidney Disease Quality of Life (KDQOL-36) with mortality and hospitalization in older adults receiving hemodialysis. BMC Nephrol 2018; 19 (1): 11.

14. Barzegar H, Jafari H, Charati JY, Esmaeili R. Relationship between duration of dialysis and quality of life in hemodialysis patients. Iran J Psychiatry Behav Sci 2017; 11 (4): 2-6 e6409.

15. Zyoud SH, Daraghmeh DN, Mezyed DO, Khdeir RL, Sawafta MN, Ayaseh NA, et al. Factors affecting quality of life in patients on haemodialysis: a cross-sectional study from Palestine. BMC Nephrol 2016; 17 (1): 1-23.

16. Zazzeroni L, Pasquinelli G, Nanni E, Cremonini V, Rubbi I. Comparison of quality of life in patients under- 
going hemodialysis and peritoneal dialysis: a Systematic Review and Meta-Analysis. Kidney Blood Press Res. 2017; 42 (4): 717-27.

17. Bohm CJ, Storsley LJ, Hiebert BM, Nelko S, Tangri N, Cheskin LJ. Impact of exercise counseling on physical function in Chronic Kidney Disease: An Observational Study. Can J Kidney Health Dis 2018; 5: 1-21.

18. Cigarroa I, Barriga R, Michéas C, Zapata LR, Soto C, Manukian T. Efectos de un programa de ejercicio de fuerza-resistencia muscular en la capacidad funcional, fuerza y calidad de vida de adultos con enfermedad renal crónica en hemodiálisis. Rev Med Chile 2016; 144 (7): 844-52.

19. Duarte PS, Ciconelli RM, Sesso R. Cultural adaptation and validation of the "Kidney Disease and Quality of Life - Short Form (KDQOL-SF ${ }^{\mathrm{TM}} 1.3$ )" in Brazil. Braz J Med Biol Res 2005; 38 (2): 261-70.

20. Chaves K, Duarte A, Vesga J. Adaptación transcultural del cuestionario KDQOL SF-36 para evaluar calidad de vida en pacientes con enfermedad renal crónica en Colombia. Rev Med 2013; 21 (2): 34-42.

21. International Physical Activity Questionnaire. Guidelines for data processing and analysis of the International Physical Activity Questionnaire (IPAQ), in Short and Long Forms; 2005. p. 1-15. Disponible en www.ipaq.ki.se

22. Barros L, Herazo Y, Aroca G. Calidad de vida relacionada con la salud en pacientes con enfermedad renal crónica. Rev Fac Med 2015; 63 (4): 641-7.

23. Seguí A, Amador P, Ramos AB. Calidad de vida en pacientes con insuficiencia renal crónica en tratamiento con diálisis. Rev Soc Esp Enferm Nefrol 2010; 13(3): 155-60.

24. Hernández A, Monguí K, Rojas Y. Descripción de la composición corporal, fuerza muscular y actividad física en pacientes con insuficiencia renal crónica en hemodiálisis en una unidad renal en Bogotá, Colombia. Rev Andal Med Deporte 2018; 11 (2): 52-6.

25. Shimoda T, Matsuzawa R, Yoneki K, Harada M, Watanabe $\mathrm{T}$, Matsumoto $\mathrm{M}$, et al. Changes in physical activity and risk of all-cause mortality in patients on maintence hemodialysis: a retrospective cohort study. BMC Nephrol 2017; 18: 154.

26. Sánchez HC, Rivadeneyra EL, Aristil ChP. Quality of life in patients on hemodialysis in a public hospital of Puebla, Mexico. AMC 2016; 20 (3): 262-70.

27. Qiu Z, Zheng K, Zhang H, Feng J, Wang L, Zhou H. Physical Exercise and Patients with Chronic Renal Failure: A Meta-Analysis. Biomed Res Int 2017; 2017: 7191826.

28. Broers NJ, Martens RJ, Cornelis T, Van der Sande F, Diederen N, Hermans M, et al. Physical Activity in End-Stage Renal Disease Patients: The Effects of Starting Dialysis in the First 6 Months after the Transition Period. Nephron Clinical Practice 2017; 137 (1): 47-56.

29. Rogan A, McCarthy K, McGregor G, Hamborg T, Evans G, Hewins S, et al. Quality of life measures predict cardiovascular health and physical performance in chronic renal failure patients. PLoS ONE 12 (9): e0183926.

30. Dziubek W, Kowalska J, Kusztal M, Rogowski Ł, Gołębiowski T, Nikifur M, et al. The Level of Anxiety and Depression in Dialysis Patients Undertaking Regular Physical Exercise Training-a Preliminary Study. Kidney Blood Press Res 2016; 41 (1): 86-98.

31. Capote LE, Argudín SR, Mora SG, Capote LP, Leonard IR, Moret YH. Evaluación de la calidad de vida relacionada con salud en pacientes en hemodiálisis periódica utilizando el KDQOL-SFTM. Medisur 2015; 13 (4): 508-16. 